\title{
Grand Challenge in Medical and Surgical Rehabilitation: From Mechanisms to Evidence Based Rehabilitation Programs
}

\author{
Christoph Gutenbrunner* \\ Department of Rehabilitation Medicine, Hannover Medical School, Hanover, Germany
}

Keywords: medical rehabilitation, surgical rehabilitation, mechanism of rehabilitation intervention, translational research, clinical trials, rehabilitation programs

\section{ASPECTS OF THE DEVELOPMENT OF REHABILITATION MEDICINE}

Looking back to the roots of modern Rehabilitation Medicine, 3 main streams can be identified.

1) The first one is the need to take care of war victims. This led to a kind of booster rehabilitation service during and after various wars (e.g., the First and Second World Wars in central Europe or the Vietnam War in The US) (1). However, past and present natural disasters have also accelerated the implementation of rehabilitation services in affected countries or regions that could be observed; an example would be in Sichuan Province in China after a deleterious earthquake in 2008. In the modern world, this stream can be seen as the root cause of post-acute and long-term rehabilitation after injury and trauma (2).

Edited and reviewed by: Paolo Capodaglio, University of Turin, Italy

${ }^{*}$ Correspondence: Christoph Gutenbrunner gutenbrunner.christoph@ mh-hannover.de

Specialty section:

This article was submitted to Medical and Surgical Rehabilitation,

a section of the journal

Frontiers in Rehabilitation Sciences

Received: 29 November 2020

Accepted: 11 December 2020

Published: 12 March 2021

Citation:

Gutenbrunner C (2021) Grand

Challenge in Medical and Surgical Rehabilitation: From Mechanisms to

Evidence Based Rehabilitation

Programs.

Front. Rehabilit. Sci. 1:634942.

doi: 10.3389/fresc.2020.634942

2) The second stream consists of care programs for children with congenital disabilities, namely deformities in the musculoskeletal system. This type of care dynamically developed in the Nineteenth century (3). Though not many historical studies exist, to some extent, the modern understanding of the provision of rehabilitation services is fundamental from a human rights perspective (4). It can be interpreted as the basis for the modern understanding of rehabilitation as a health strategy to reduce disability as a lived experience of persons with impairments $(2,5)$.

3) The third stream originates from the tradition of health resort medicine and treatment concepts that use balneological and physical modalities (6). These types of treatment have had a central role in the treatment of chronic health conditions since the Nineteenth century and were then implemented in the modern social and health insurance systems (7). It was developed in central, southern, and eastern Europe; however, some of the traditions were used in the UK and the US. In Europe, it became the basis for the rehabilitation of people with chronic health conditions and to prevent the need for health-related early pension payment. Conceptually, this stream was the basis of the development of rehabilitation concepts for persons with chronic and progressive health conditions (8).

Taking these streams together, today there is strong agreement that rehabilitation must be available during all phases (acute, post-acute, and long-term care) and at all levels (primary, secondary, and tertiary level) of health care (8). It has been defined as an essential part of Universal Health Coverage (9) and has been labeled "the health strategy of the twenty-first century" (2). 
These historical and conceptual reflections on rehabilitation should not lead to underestimation of the importance of health interventions that target the improvement of body functions and structures, activities, and participation. From a scientific perspective, many aspects have to be investigated (10). This includes biomolecular mechanisms of the repair and healing processes, the mechanisms of action of applied physical and chemical modalities, translational research, clinical trials on effects and effectiveness of rehabilitation interventions, and the applicability of rehabilitation programs in healthcare settings. Such research has, apart from its generic aspects, a conditionspecific focus, and it is of major importance to a wide range of medical and surgical conditions.

\section{MAIN STREAMS AND GAPS IN BASIC AND CLINICAL REHABILITATION SCIENCES}

Looking at the topics published in scientific journals in the field of rehabilitation medicine, it becomes evident that diseases of the nervous and musculoskeletal systems as well as cardiac and respiratory conditions are predominant areas of research (11). The same profile results, if the abstract submissions to scientific congresses in the rehabilitation field are analyzed (12). Assuming that health-related rehabilitation measures aim at reducing impairments and facilitate functioning independent of the underlying health condition, the focus of rehabilitation medicine must be much broader. A few examples may illustrate this:

- Mental disorders, including depression and anxiety, are frequent health conditions and have a high impact on participation in society and vocational performance $(13,14)$. Persons with mental disorders have a high need for a wide range of rehabilitation interventions, such as psychotherapy, physical exercise, behavioral treatments, social counseling, and medications. Not much has been published on the outcomes of such multimodal rehabilitation programs, the effects of single interventions (incl. dosage), the (synergistic) effects of combined treatments, and the duration of rehabilitation interventions. Additionally, knowledge of predictors, age- and gender-specific effects, and the adherence of effects need further elucidation.

- Another field that has not yet sufficiently investigated is rehabilitation for children with specific health conditions (with the possible exceptions of cerebral palsy and juvenile rheumatoid arthritis). The gaps are obvious when looking at children with cancer and mental disorders $(15,16)$. With regard to mechanisms, it should be taken into account that repair mechanisms and the immediate effects of interventions may differ significantly with age and developmental phase. Last but not least the inclusion of parents and other family members into rehabilitation programs must be the focus of research (17).

- Bladder and prostate surgery are other examples of the need for surgical rehabilitation. A number of post-surgical symptoms have a high impact on the patient's quality of life (18). These range from incontinence, external urination systems, sexual dysfunction, fatigue, and mental health problems (19). This demonstrates the need for highly specialized rehabilitation approaches. The effectiveness and efficacy of such interventions must be evaluated. This is also the case for treatment combinations. Another issue from the point of view of a clinician is rehabilitation intervention for sexual functions. Last but not least, research on the impact of surgical techniques on long-term functional outcomes should be investigated more.

- Another recent topic for rehabilitation medicine research is long-term impairment following SARS-CoV-2 infection (addressed as "post-COVID-19-Syndrome" or "long-term COVID-19”) (20). Besides respiratory dysfunction, various symptoms have been observed, such as disturbances of smell and taste, dysfunctions in cognition, memory, and motor coordination, muscular pain, fatigue, and depression (21). In principle, all these symptoms are available, but we do not know if this applies to this type of pathology or if any specific strategies must be developed. As it is a new disease, research is needed to elucidate the frequency and severity of symptoms, its impact on participation, and the effectiveness of interventions.

Other conditions as well as rehabilitation of persons with multimorbidity, rehabilitation for old people and patients with different types of cancer could have been taken as examples.

With regard to mechanisms, the knowledge on neuroplasticity and brain repair, training of muscle functions, and influence on tropho-trophic tissues of the musculoskeletal system as well on cardiovascular, respiratory, and metabolic functions and its relevance for rehabilitation approaches have grown dramatically during the last decades; other pathophysiological and repair mechanisms have received much less attention. Examples for this are possible influences on the biomolecular mechanisms of wound healing, mechanisms of pain chronification, and the immune response.

Another issue is that rehabilitation research-similar to the implementation of rehabilitation programs-focuses on the most frequent health conditions, such as back pain, osteoarthritis, diabetes, COPD, myocardial infarction, etc. The development of rehabilitation programs for rare diseases and the research about it is extremely scarce. From an ethical perspective and with regard to scientific relevance, this should be changed (22), and research should be facilitated. This must include an assessment of rehabilitation needs too.

\section{FUTURE STEPS IN MEDICAL AND SURGICAL REHABILITATION}

The abovementioned examples demonstrate that the field of medical and surgical rehabilitation needs much more attention in research. To conceptualize such fields, more systematic consideration is needed.

For efficient rehabilitation, goal setting has an important role (23). For a systematic goal-setting process that must be based both on the nature and extent of limitations in functioning as well as the patients' perspectives and individual goals, an appropriate assessment is crucial (24). Many assessment tools, 
including testing of body functions and activities as well as checklists and questionnaires, are available $(25,26)$. These mostly include condition-specific and functioning-specific aspects. Most condition-specific tools concentrate on frequent rehabilitation diagnoses, such as disorders of the musculoskeletal and nervous system as well as cardiovascular and lung pathologies. There is a need for the development of condition-specific tools or modules for less frequent pathologies. Limb amputation, surgical conditions, and even the post-COVID-19 syndrome are examples.

In many clinical fields, the treatment strategies and intervention emerge very dynamically. This is the case for surgical techniques, pharmacological treatments, and cognitivebehavioral approaches. It is extremely important to investigate how this influences rehabilitation strategies and-on the other hand-how rehabilitation needs may be modified. Some examples for that are the implementation of endo-exo-prothesis after limb amputation, biologicals in the treatment of rheumatoid arthritis, organ transplantation surgery, and others. Furthermore, rehabilitation technology and assistive devices as well as robotics and it-approaches are developing fast. Both aspects must be considered in designing rehabilitation approaches and must be investigated in clinical trials.

Condition-specific rehabilitation programs may distract the attention on so-called non-specific symptoms that are frequently linked to the condition that is seen as an indication for rehabilitation. One example is the phenomenon of fatigue that can be described both as physical and mental fatigue (27). Other such symptoms may be sleep disturbance, widespread pain, thermal discomfort, and a depressed mood. These symptoms or dysfunctions can be observed in patients with diverse pathologies, e.g., cancer (including post-treatment phase), infectious diseases, musculoskeletal pain disorders, metabolic disorders, and others. Recently, it has been described that postCOVID-19 syndrome to a high extent also includes suchs

\section{REFERENCES}

1. Atanelov L, Stiens SA, Young MA. History of physical medicine and rehabilitation and its ethical dimensions american medical association. $J$ Ethics. (2015) 17:568-74. doi: 10.1001/journalofethics.2015.17.6.mhst1-1506

2. Stucki G, Bickenbach J, Gutenbrunner C, Melvin J. Rehabilitation: the health strategy of the 21st century. J Rehabil Med. (2018) 50:309-16. doi: 10.2340/16501977-2200

3. Fichtner HJ. From the care of cripples to the rehabilitation of the physically handicapped. Rehabilitation. (1985) 24:113-5.

4. United Nations. Convention on the Rights of Persons with Disabilities. New York, NY: United States (2006).

5. Krug E, Cieza A. Strengthening health systems to provide rehabilitation services. Can J Occup Ther. (2017) 84:72-75. doi: 10.1177/0008417417705853

6. European Physical and Rehabilitation Medicine Bodies Alliance. White book on physical and rehabilitation medicine (PRM) in Europe. Chapter 4. History of the specialty: where PRM comes from. Eur J Phys Rehabil Med. (2018) 54:186-97. doi: 10.23736/S1973-9087.18.05147-X

7. Simon P. The development of legal premises in the rehabilitation of the physically handicapped from Bismarck to today - a brief overview. Rehabilitation. (1985) 24:131-3.

8. Gutenbrunner C, Nugraha B. Physical and rehabilitation medicine: responding to health needs from individual care to service provision. symptoms (21). From the point of view of pathomechanisms, responses of the immune system to the primary pathology may play a role (28). On the other hand, most non-specific symptoms are related to autonomous regulation, namely, the hypophysis-pituitary-adrenal axis (29). Last but not least, specific hormonal dysregulation (e.g., in cancer) and mediators (e.g., in chronic widespread pain) are involved (30-32). As non-specific symptoms may have a major impact on quality of life and hinder participation, rehabilitation must focus on these. Both, studies on mechanisms as well as on programs and outcomes are required. Related to this, studies on biomolecular mechanisms as well as on adaptation and repair mechanisms need to be performed and integrated into a concept of translational research. This also must include mechanisms of treatment effects both as single intervention and as serial applications.

Last but not least the organization of rehabilitation services and their interfaces with other health services may significantly influence service quality and outcomes (33). Here, it is also important to reflect and investigate what specific requirements rehabilitation services must fulfill in order to meet the needs of medical and surgical conditions (e.g., cancer, mental disorders, organ transplantation, and others).

All in all, it can be seen that the whole spectrum of rehabilitation research "from cell to society" (10) is of relevance for a scientific approach toward improvements in rehabilitation for medical and surgical conditions. Taking such a broad approach will need careful interpretation and linking of different aspects. It will contribute to better provision of care and outcomes for patients in need of rehabilitation.

\section{AUTHOR CONTRIBUTIONS}

The author confirms being the sole contributor of this work and has approved it for publication.

Eur J Phys Rehabil Med. (2017) 53:1-6. doi: 10.23736/S1973-9087.17. 04657-3

9. Mills JA, Marks E, Reynolds T, Cieza A. Chapter 15: rehabilitation: essential along the continuum of care. In: Jamison DT, Gelband H, Horton S, editors. Disease Control Priorities: Improving Health and Reducing Poverty. 3rd ed. Washington, DC: The International Bank for Reconstruction and Development; The World Bank (2017). doi: 10.1596/978-1-4648-05271_ch15

10. Stucki G, Reinhardt JD, Grimby G, Melvin J. Developing "human functioning and rehabilitation research" from the comprehensive perspective. J Rehabil Med. (2007) 39:665-71. doi: 10.2340/16501977-0136

11. Gutenbrunner C, Nugraha B. Introduction to the scientific background of Physical and Rehabilita-tion Medicine. The scientific basis of physical and rehabilitation medicine. The scope of physical and rehabilitation medicine. J Int Soc Phys Rehabil Med. (2019) 2:102-3. doi: 10.4103/jisprm.jisprm_23_19

12. Nugraha B, Paternostro-Sluga T, Schuhfried O, Stucki G, Franchignoni F, Latif LA, et al. Evaluation of the topic list used in two world congresses (2015 ad 2016) in physical and rehabilitation medicine. J Rehabil Med. (2017) 49:469-74. doi: 10.2340/16501977-2232

13. Rössler W. Psychiatric rehabilitation today: an overview. World Psychiatry. (2006) 5:151-7.

14. Pratt CW, Gill KJ, Barrett NB, Roberts MM. Psychiatric Rehabilitation. 3rd ed. Amsterdam: Elsevier Inc (2013). doi: 10.1016/B978-0-12-387002-5.00005-6 
15. Whitney R, Hilton CL. Intervention effectiveness for children and youth. Am J Occup Ther. (2013) 67:154-65. doi: 10.5014/ajot.2013.008557

16. Kerbl R, Sperl W, Strassburg HM, Pettoello-Mantovani M, Ehrich J. Overview of habilitation and rehabilitation for children and adolescents in Europe. $J$ Pediatr. (2016) 172:233-5. doi: 10.1016/j.jpeds.2015.12.078

17. King G, Williams L, Hahn Goldberg S. Family-oriented services in pediatric rehabilitation: a scoping review and framework to promote parent and family wellness. Child Care Health Dev. (2017) 43:334-47. doi: 10.1111/cch.12435

18. Kim TB, Kim CH, Kim KT, Yoon SJ, Chung KJ. Urology as rehabilitation medicine: a literature review. J Exerc Rehabil. (2018) 14:322-6. doi: 10.12965/jer.1836222.111

19. Köhler N, Friedrich M, Gansera L, Holze S, Thiel R, Roth S, et al. Psychological distress and adjustment to disease in patients before and after radical prostatectomy. Results of a prospective multi-centre study. Eur J Cancer Care. (2014) 23:713-820. doi: 10.1111/ecc.12186

20. Yelin D, Wirtheim E, Vetter P, Kalil AC, Bruchfeld J, Runold M, et al. Longterm consequences of COVID-19: research needs. Lancet. (2020) 20:P11157. doi: 10.1016/S1473-3099(20)30701-5

21. Lopez M, Bell K, Annaswamy T, Juengst S, Ifejika N. COVID-19 guide for the rehabilitation clinician: a review of nonpulmonary manifestations and complications. Am J Phys Rehabil Med. (2020) 99:669-73. doi: 10.1097/PHM.0000000000001479

22. Binkiewicz-Glińska A, Ruckermann-Dziurdzińska K. Pediatric rehabilitation in children with rare diseases-preliminary report. Dev Period Med. (2015) 19:516-8.

23. Wade DT. Goal setting in rehabilitation: an overview of what, why and how. Clin Rehabil. (2009) 23:291-5. doi: 10.1177/0269215509103551

24. Turner-Stoke L. Goal attainment scaling (GAS) in rehabilitation. A practical guide. Clin Rehabil. (2009) 23:362-70. doi: 10.1177/0269215508101742

25. Gutenbrunner C, Cantista P, Ceravolo MG, Christodoulou N, Delarque A, Kiekens C, et al. White Book on physical and rehabilitation medicine in Europe. Chapter 7: the clinical field of competence: PRM in practice. Eur J Phys Rehabil Med. (2018) 54:230-60. doi: 10.23736/S1973-9087.18. 05151-1
26. Gimigliano F, Iolascon G. Physical and rehabilitation medicine clinical scope: medical diagnostic tools. The scope of physical and rehabilitation medicine. $J$ Int Soc Phys Rehabil Med. (2019) 2:35-40. doi: 10.4103/jisprm.jisprm_11_19

27. Bower JE. Cancer-related fatigue: mechanisms, risk factors, and treatments. Nat Rev Clin Oncol. (2014) 11:597-609. doi: 10.1038/nrclinonc.201 4.127

28. Calvo M, Dawes JM, Bennett DLH. The role of the immune system in the generation of neuropathic pain. Lancet Neurol. (2012) 11:629642. doi: 10.1016/S1474-4422(12)70134-5

29. DeMorrow S. Role of the hypothalamic-pituitary-adrenal axis in health and disease. Int J Mol Sci. (2018) 19:986-71. doi: 10.3390/ijms1904 0986

30. Maggio M, Cattabiani C, Lauretani F, Ferrucci L, Luci M, Valenti G, et al. The concept of multiple hormonal dysregulation. Acta Biomed. (2010) 81:19-29.

31. Blundon MA, Dasgupta S. Metabolic dysregulation controls endocrine therapy-resistant cancer recurrence and metastasis. Endocrinology. (2019) 160:1811-2. doi: 10.1210/en.2019-00097

32. McHugh JM, McHugh WB. Pain: neuroanatomy, chemical mediators, and clinical implications. Clin Issues Adv Pract Acute Crit Care. (2000) 11:16878. doi: 10.1097/00044067-200005000-00003

33. Gutenbrunner C, Nugraha B. Decision making in evidence-based practice in rehabilitation medi-cine: proposing a fourth factor. Am J Phys Med Rehabil. (2020) 99:436-40. doi: 10.1097/PHM.0000000000001394

Conflict of Interest: The author declares that the research was conducted in the absence of any commercial or financial relationships that could be construed as a potential conflict of interest.

Copyright (C) 2021 Gutenbrunner. This is an open-access article distributed under the terms of the Creative Commons Attribution License (CC BY). The use, distribution or reproduction in other forums is permitted, provided the original author(s) and the copyright owner(s) are credited and that the original publication in this journal is cited, in accordance with accepted academic practice. No use, distribution or reproduction is permitted which does not comply with these terms. 